\title{
Damping and Slipping Behavior of Highly Entangled Polyisobutylene
}

\author{
Katsuyuki Yoshikawa, Fei Teng, Takumi KaBeYA, Jun-ichi HorinaKA, and Toshikazu TAKIGAWA ${ }^{\dagger}$ \\ Department of Material Chemistry, Kyoto University, \\ Nishikyo-ku, Kyoto 615-8510, Japan \\ (Received : September 17, 2015)
}

\begin{abstract}
We examine damping and slipping behavior of highly entangled polyisobutylene (PIB). Two types of PIB samples were prepared for comparison: One is PIB melts stuck with the metal plates of rheometer with adhesive, and another is the melts without adhesive. Comparison of the damping and the stress-strain behavior of the two types of samples gives an evidence of slipping for the samples without adhesive. Together with the data for another grade of PIB, it is suggested that a critical value for slipping does not depends on the molecular weight and lies around 0.2 MPa.
\end{abstract}

Key Words: Polyisobutylene / Slip / Step strain / Stress relaxation

As well known, the relaxation modulus of polymer liquids at long times can be written in the separable form as ${ }^{1,2)}$

$$
G(t, \gamma)=G(t) h(\gamma)
$$

where $G(t, \gamma)$ is the relaxation modulus at time $t$ and strain $\gamma$, and $G(t)$ is the linear relaxation modulus, $h(\gamma)$ being the damping function. For highly entangled linear polymers with narrow molecular weight distributions, the damping function in shear usually show a stronger strain dependence than the Doi-Edwards (DE) theory ${ }^{1)}$ predicts. The origin of the strong damping is considered to be due to the deformation-induced phase separation. ${ }^{3)}$ Originally, this was proposed for the shear deformation but must be applicable to the uniaxial elongation, because the possibility of phase separation depends only on the (pseudo-) strain energy function, which can generally be described by the strain invariants, for the polymer melts.

We have reported the damping behavior of highly entangled high density polyethylene (HDPE) in shear and in uniaxial elongation. ${ }^{4,5)}$ These show that damping function in uniaxial elongation does not show such strong damping even if the damping function in shear shows a strong damping. ${ }^{4,5)}$

For highly entangled polyisobutylene (PIB) melts, the similar behavior has been observed. ${ }^{6)}$ In addition, the effect of slipping at the interface between the metal plate of rheometer and the sample surface on the damping behavior has been examined for the PIB sample. ${ }^{6}$ The PIB sample stuck on the

$\dagger$ Corresponding author

E-mail: takigawa.toshikazu.7u@kyoto-u.ac.jp metal plates by using an adhesive ("with-adhesive" sample) shows a stress development as elastomers do in the course of step-like strain application process for stress relaxation. ${ }^{6}$ On the other hand, the PIB sample without adhesive ("withoutadhesive" sample) moves on the same path as "with-adhesive" in the small strain region but then shows branching in the large strain region. ${ }^{6}$ This bifurcation is considered to be a critical point of slipping for the PIB sample. ${ }^{6}$

The PIB sample employed above was just one grade, so that we cannot clarify whether or not the critical stress becomes a material constant (if the metal species of the fixture is specified). In this study, the damping and stress growth behavior of two PIB samples (PIB-1 and PIB-3), which are different from the sample used in the previous study (we designate here the previous PIB sample as PIB-2), are examined in shear. By combining obtained data with those for PIB-2 how the molecular weight of PIB affects the critical stress for slipping is examined.

PIB-1 and PIB-3 were purchased from Sigma-Aldrich Co., USA. The number-average and weight-average molecular weights $\left(M_{\mathrm{n}}\right.$ and $M_{\mathrm{w}}$, respectively), and the ratio $M_{\mathrm{w}} / M_{\mathrm{n}}$ for the PIB samples are listed in Table I, together with those for PIB-2. Since the molecular weight between entanglements $\left(M_{\mathrm{e}}\right)$ for PIB is reported to be $8900,{ }^{7)}$ we calculated the number of entanglements per chain for the three PIB samples by $M_{\mathrm{n}} / M_{\mathrm{e}}$. These values are also listed in Table I. According to the Osaki's criterion, ${ }^{2)}$ PIB-2 and PIB-3 are categorized in to the highly entangled systems because the number of entanglements per chain is larger than 50 for the PIBs. The 
value of $M_{\mathrm{n}} / M_{\mathrm{e}}$ for PIB-1 is smaller than 50, and thus PIB-1 is categorized into the moderately entangled systems.

Disk-shaped samples for rheological measurements were made by using a compression-molding machine: PIB-1 was compression-molded at $50{ }^{\circ} \mathrm{C}$ for $1 \mathrm{~h}$ and PIB-3 was done at $80{ }^{\circ} \mathrm{C}$ for $24 \mathrm{~h}$. Rheological measurements were carried out at $50{ }^{\circ} \mathrm{C}$ by using an ARES rheometer (TA Instruments, USA). The fixture used was parallel-plate with a diameter of $8 \mathrm{~mm}$. For PIB-3, two types of sample settings into metal fixture were made: One is the sample setting with adhesive, and another is that without adhesive. Aron Alpha ${ }^{\circledR}$ (Toagosei, Japan) was used as an adhesive for sticking the sample surface on the metal plate of the rheometer. For PIB-1 only the setting of "without adhesive" was employed, because good adhesion by the adhesive was not realized for PIB-1.

Stress relaxation were measured at $50{ }^{\circ} \mathrm{C}$. As the stress relaxation mode on ARES provided the stress and strain data in the strain growth process to the pre-set value, the stress and strain values in the transient state were also analyzed to see the occurrence of slipping. Dynamic viscoelasticity of the PIB samples before and after stress relaxation measurements was measured, and no marked change by stress relaxation experiments was confirmed. In the analysis of the damping and slipping behavior, the data for PIB-2 samples with and without adhesive were used together with those for PIB-1 and PIB-3 obtained in this study.

Figure 1 shows the stress relaxation curves of PIB-3 without and with adhesive: Panel (a) contains the curves for "without adhesive" and panel (b) corresponds to the curves for "with adhesive". The curves for "without adhesive" and "with adhesive" are very similar in shape, but are different in damping behavior. For "without adhesive" we were able to measure the relaxation modulus at $\gamma \leq 1.0$. For "with adhesive" the measured region was limited to $\gamma \leq 0.5$ due to overload of system at large strains. Nevertheless, "without adhesive" clearly shows stronger damping than "with adhesive", which is consistent with the results of PIB-2. ${ }^{6}$ Concerning the position of the linear relaxation modulus, which is here assumed to be $G(t, \gamma)$ at $\gamma=0.1$, is 1.15 times higher than that for "without adhesive", probably due to the effect of adhesion. The similar enhancement of modulus by adhesion is also

Table I. Molecular characteristics of PIB samples.

\begin{tabular}{ccccc} 
& $M_{\mathrm{n}}$ & $M_{\mathrm{w}}$ & $M_{\mathrm{w}} / M_{\mathrm{n}}$ & $M_{\mathrm{n}} / M_{\mathrm{e}}$ \\
\hline PIB-1 & $2.0 \times 10^{5}$ & $5.0 \times 10^{5}$ & 2.5 & 22 \\
PIB-2 & $6.0 \times 10^{5}$ & $1.0 \times 10^{6}$ & 1.7 & 67 \\
PIB-3 & $3.1 \times 10^{6}$ & $4.7 \times 10^{6}$ & 1.5 & 350 \\
\hline
\end{tabular}

observed for PIB-2. ${ }^{6}$ Although we do not show the figure, the damping behavior of "without adhesive" of PIB-1 was not so strong as that of PIB-3.

Figure 2 shows the damping function $h(\gamma)$ plotted against $\gamma$ for the PIBs. This figure contains the data points for PIB-2 obtained in the previous study. ${ }^{6}$ The DE prediction with the independent alignment approximation is also drawn in this figure. For PIB-2 and PIB-3, little deviation from the DE predictions is observed for "with adhesive" (although the number of data points are rather small) but marked deviation occurs for the "without adhesive" samples. The damping function of "without adhesive" of PIB-1 agrees well with the DE prediction up to $\gamma=0.7$, but at $\gamma=1.0$ a slight downward shift (or, a little stronger damping) is observed. The stronger damping is observed only for the "without adhesive" samples.

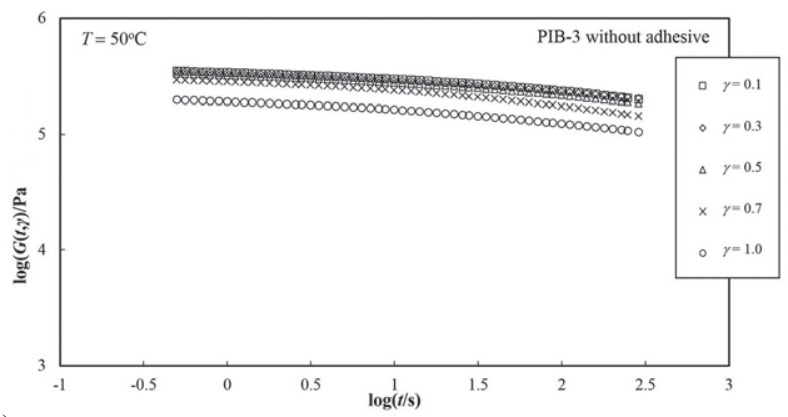

(a)

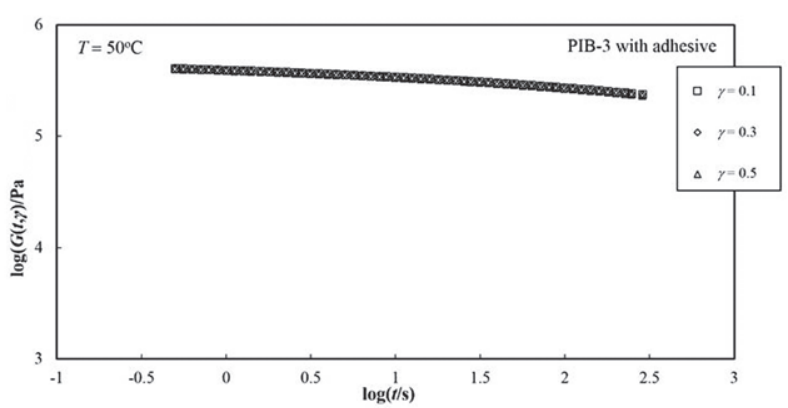

(b)

Fig. 1. (a) Relaxation modulus at time $t$ and strain $g(t, \gamma)$ for PIB-3 without adhesive.

(b) Relaxation modulus at time $t$ and strain $\gamma G(t, \gamma)$ for PIB-3 with adhesive.

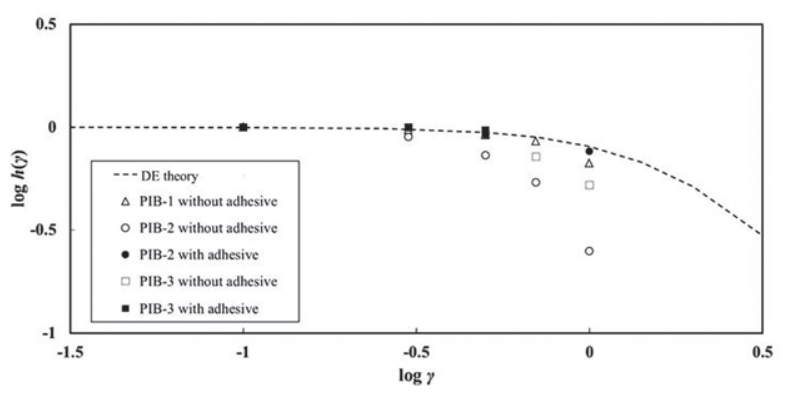

Fig. 2. Damping function $h(\gamma)$ plotted against strain $\gamma$ for PIB samples. 


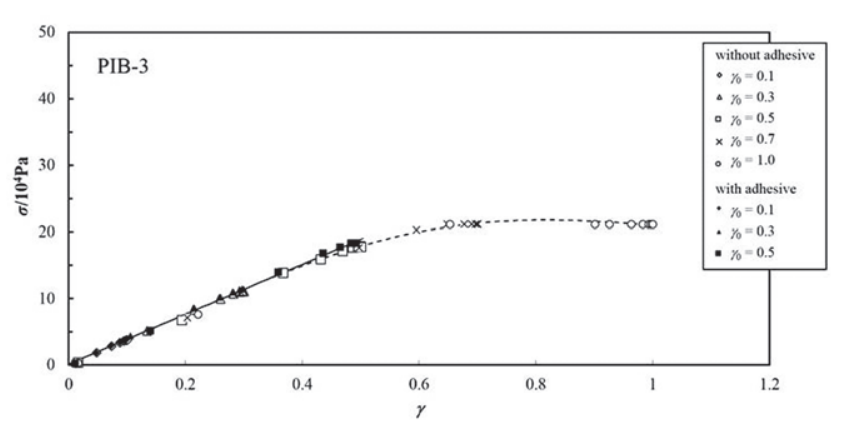

Fig. 3. Stress $(\sigma)$-strain $(\gamma)$ curves in the course of strain application process for stress relaxation for PIB-3.

This appears to indicate that the stronger damping originates from slipping.

Figure 3 shows the stress-strain curves for "with adhesive" and "without adhesive" of PIB-3 obtained in the course of stress relaxation experiments. Since ARES originally provides the stress and strain data as a function of time, the stress is re-plotted as a function of strain in the transient state to the preset strains (or, target strains). The data points used here are limited; only those in the time domain of $t<100 \mathrm{~ms}$ in each stress relaxation experiment are included. It should be noted here that the rise time for strain (control parameter on ARES) was fast and the target value of strain was reached in $3 \mathrm{~ms}$ just after starting. ${ }^{6}$ As stated previously, the linear modulus of "with adhesive" is 1.15 times higher than that of "without adhesive", the stress values of "with adhesive" are divided by 1.15. At $\gamma$ $<\sim 0.45$, the stress-strain curves for "without adhesive" and "with adhesive" become identical, and they remain linear in this region. For "with adhesive" the linear relation continues up to the upper limit of $\gamma=0.5$. This is due to overload of system at $\gamma>0.5$ (see Figure 1(b)). The stress-strain curve for "without adhesive" is prepared with the data in the region of $0<\gamma<1.0$. This curve appears to branch from the curve for "with adhesive" at $\sim 0.45$, then extending almost horizontally. These suggest that slipping initiates at $\gamma=\sim 0.45$ for "without adhesive". The stress value at this branching point is estimated to be $0.15 \mathrm{MPa}$ and the saturated value of stress to be $0.2 \mathrm{MPa}$, indicating that these are very close to each other.

In Figure 4 the stress-strain curves of all PIB samples are summarized. For PIB-3 the same curves as shown in Figure 3 are re-plotted in this figure. Although the curves for PIB-2 have already been published, ${ }^{6)}$ they are again shown here for comparison. For PIB-1 we have only "without adhesive", and the curve shows a gradual rise with strain compared with those of PIB-2 and PIB-3. The curve of PIB-1appears to show leveling-off behavior around the larger strain limit $(\gamma>0.9)$, and this plateau value agrees well with that for "without

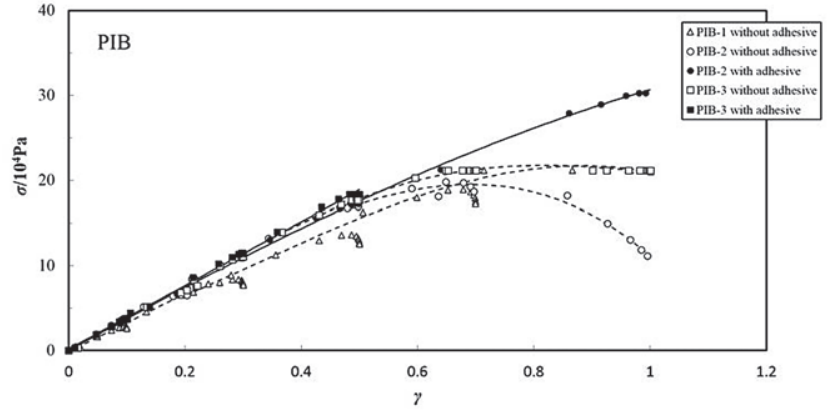

Fig. 4. Stress $(\sigma)$-strain $(\gamma)$ curves in the course of strain application process for stress relaxation for PIB samples.

adhesive" of PIB-3 (0.2MPa). We cannot estimate an actual stress value at the branching point for PIB-1, but the stress value must be not so far from the leveling-off value (0.2 $\mathrm{MPa})$ as in the case of PIB-3. A marked difference is observed on the curves of "with adhesive" and "without adhesive" for PIB-2." The stress monotonically increases with increasing strain for "with adhesive". The curve of "without adhesive" moves on the same line as that of "with adhesive" at small strains, and then branching occurs at $\gamma=\sim 0.5$. After branching the curve shows a maximum, unlike the case of PIB-3. The stress at the branching point is $\sim 0.17 \mathrm{MPa}$. This is very close to the value for PIB-3 (0.15 MPa).

When a critical stress is defined as the stress at the branching point, the critical stress characterized the onset of slipping. This critical stress is obtained to be 0.17 for PIB-2 and $0.15 \mathrm{MPa}$ for PIB-3. Unfortunately, the critical stress for PIB-1 is unknown at present, but the value may be replaced by the leveling-off value of 0.2 MPa. By comparing these values, it is suggested that the critical stress value for slipping of PIB (more exactly, with the metal surface of ARES, namely, stainless steel) stays constant and no molecular weight dependence exists for the critical value.

\section{REFERENCES}

1) Doi M, Edwards SF, The Theory of Polymer Dynamics, Clarendon, Oxford, 1986.

2) Osaki K, Rheol Acta, 32, 429 (1993).

3) Marrucci G, Grizzuti N, J Rheol, 27, 433 (1983).

4) Kabeya T, Yoshikawa K, Horinaka J, Takigawa T, J Soc Matr Sci Jpn, 64, 47 (2015).

5) Kabeya T, Yoshikawa K, Horinaka J, Takigawa T, Nihon Reoroji Gakkaishi, 43, 11 (2015).

6) Yoshikawa K, Kabeya T, Horinaka J, Takigawa T, Nihon Reoroji Gakkaishi, in press.

7) Ferry JD, Viscoelastic Properties of Polymers, 3rd ed, Wiley, New York, 1980. 